\title{
Coherent control in atomic chains: To trap and release a traveling excitation
}

\author{
R. Gutiérrez-Jáuregui $~^{*}{ }^{*}$ and A. Asenjo-Garcia ${ }^{\dagger}$ \\ Department of Physics, Columbia University, New York, New York 10027, USA
}

(Received 27 April 2021; revised 27 July 2021; accepted 23 December 2021; published 1 February 2022)

\begin{abstract}
We introduce a protocol for dynamical dispersion engineering in an atomic chain formed by an ordered array of multilevel atoms with subwavelength lattice constant. This chain supports dark states that are protected from dissipation and can be understood as spin waves traveling along the array. By using an external control field with a spatially varying elliptical polarization we correlate internal and external degrees of freedom of the array in a controllable way. The coherent control over atomic states translates into control over the group velocity of the spin waves. A traveling excitation can be stored and released without dissipation by adiabatically changing the control field amplitude. This protocol is an alternative to the more conventional electromagnetically induced transparency and exemplifies the rich physics born of the interplay between coherent control and correlated decay.
\end{abstract}

DOI: 10.1103/PhysRevResearch.4.013080

\section{INTRODUCTION}

Recent years have witnessed a renewed interest in the optical response of atomic arrays with subwavelength interatomic distances. This interest is driven by an exquisite control acquired over atomic positions that allows for arbitrary shapes to be constructed from single-atom components [1-6]. As the collective decay of an array is determined by the interference between radiative paths of individual atoms, its optical response is intimately connected to the underlying geometry. Atoms arranged in a two-dimensional array have been show to act as a subradiant mirror [6] while one-dimensional (1D) chains and rings have been proposed to construct lossless atomic waveguides [7,8] and cavities [9]. While these responses showcase the potential of ordered arrays as versatile light-matter interfaces, an extension towards dynamical engineering remains challenging.

Control over the internal degrees of freedom in lightmatter interfaces provides an attractive tool to manipulate their response in real time. A striking example is that of electromagnetically induced transparency (EIT) [10-13], where laser pulses traveling inside a disordered atomic gas can be slowed down significantly. In EIT, a transparency window is opened into an otherwise optically dense medium by means of an external control field that generates a dark state due to destructive interference. Light propagates through the medium as a polariton [14] whose speed is reduced as the excitation is predominantly transferred to the dark state of the atomic gas. Moreover, this polariton can be brought to a standstill by using a time-varying control field $[15,16]$.

\footnotetext{
*r.gutierrez.jauregui@gmail.com

†ana.asenjo@columbia.edu
}

Published by the American Physical Society under the terms of the Creative Commons Attribution 4.0 International license. Further distribution of this work must maintain attribution to the author(s) and the published article's title, journal citation, and DOI.
Here, we explore the interplay between coherent control and correlated decay in the most simple arrangement, a 1D atomic array, and present a protocol for dynamical dispersion engineering that allows for trapping and releasing single traveling excitations. We use an external control field that is far-detuned from the excited states of atoms with a three-level $V$ configuration. The control field displays a subwavelength polarization gradient that modifies the dipole-dipole interactions between the atoms and effectively couples their internal (spin) and external (position) degrees of freedom. Coherent control over the internal states is translated into control over the group velocity of an excitation that propagates through the array. Adiabatic changes of the helicity of the control field allow us to slow down, trap, and reverse the direction of traveling excitations, without being limited by single-atom spontaneous emission.

\section{CONTROLLED RESPONSE THROUGH A FAR-DETUNED FIELD}

The proposed setup, sketched in Fig. 1, consists of a chain of lattice constant $a$ where each atom is characterized by its position $z_{n}$ and is assumed to have a ground state $\left|g^{n}\right\rangle$ and three excited states $\left|e_{s}^{n}\right\rangle(s=\{0, \pm\})$. The chain is illuminated by a control field composed of two counterpropagating plane waves of electric-field amplitudes $E_{+}$and $E_{-}$that share the same frequency $\omega_{c}$ but have counter-rotating circular polarizations $\mathbf{e}_{ \pm}$. Their superposition creates a standing wave of elliptical polarization and constant ellipticity throughout the chain [17],

$$
\mathbf{E}_{c}(\mathbf{z}, t)=e^{-i \omega_{c} t}\left\{\left(E_{+}+E_{-}\right) \mathbf{e}_{x}^{\prime}+i\left(E_{+}-E_{-}\right) \mathbf{e}_{y}^{\prime}\right\}+\text { c.c. },
$$

whose axes $\mathbf{e}_{x, y}^{\prime}$ rotate around the chain axis,

$$
\begin{aligned}
\mathbf{e}_{x}^{\prime} & =\cos k_{c} z \mathbf{e}_{x}-\sin k_{c} z \mathbf{e}_{y}, \\
\mathbf{e}_{y}^{\prime} & =\sin k_{c} z \mathbf{e}_{x}+\cos k_{c} z \mathbf{e}_{y} .
\end{aligned}
$$

We first discuss how the control field induces a positiondependent dipole moment in the atoms due to its polarization 


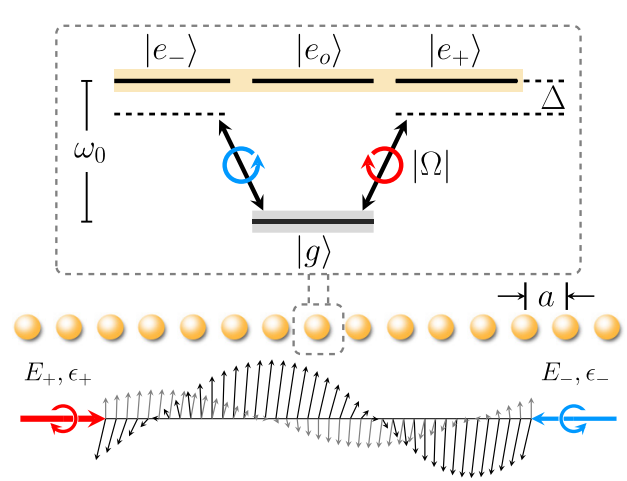

FIG. 1. An atomic chain of lattice constant $a$ is driven by a fardetuned control field with periodic polarization gradient. The field consists of two counterpropagating circularly polarized plane waves that form a standing wave of elliptical polarization whose axes rotate along the chain axis (black and gray arrows) and induces a positiondependent dipole moment by coupling ground $|g\rangle$ and excited $\left|e_{ \pm}\right\rangle$ states. The coupling strength depends on the Rabi frequency $|\Omega|$ and detuning $\Delta$ with a relative phase that depends on the atomic position. The field enables control over the dispersion relation of dark states that emerge in the chain for lattice constants $a<\lambda_{0} / 2\left(\lambda_{0}\right.$ being the atomic transition wavelength).

gradient. Each atom couples to this field via the dipolar interaction $\hat{\mathbf{d}}^{(n)} \cdot \mathbf{E}_{\mathrm{c}}\left(z_{n}\right)$ with $\hat{\mathbf{d}}^{(n)}$ being the electric-dipole moment operator and $\mathbf{E}_{\mathrm{c}}\left(z_{n}\right)$ the field amplitude evaluated at the atomic position $z_{n}$. The coupling strength $\hbar \Omega=$ $\sum_{s} d_{s} E_{s}\left(z_{n}\right)$ is determined by the electric-dipole matrix elements between states $g$ and $e_{s}$, denoted by $d_{s}$, and the projection of the field along their respective orientations. With the control field far-detuned from the atomic transition frequency $\omega_{0}\left(\Delta \gg|\Omega|\right.$, with $\left.\Delta=\omega_{0}-\omega_{c}\right)$, its effect on the $n$th atom is accurately described by the Hamiltonian [18-20]

$$
\begin{aligned}
\hat{\mathcal{H}}_{\mathrm{eff}}^{(n)}= & \frac{\hbar}{2}(\Delta+\delta)\left(\sum_{s= \pm} \hat{\sigma}_{s s}^{(n)}-\hat{\sigma}_{g g}^{(n)}\right) \\
& -\sum_{s= \pm} \frac{\hbar \delta}{4}(1-s \cos \theta) \hat{\sigma}_{s s}^{(n)} \\
& +\frac{\hbar \delta}{4} \sin \theta\left(e^{-2 i k_{c} z_{n}} \hat{\sigma}_{+-}^{(n)}+e^{2 i k_{c} z_{n}} \hat{\sigma}_{-+}^{(n)}\right),
\end{aligned}
$$

plus an additional term accounting for the state $\left|e_{0}^{n}\right\rangle$, which remains decoupled and is ignored throughout. In this expression, the operator $\hat{\sigma}_{s s^{\prime}}^{(n)}=\left|e_{s}^{n}\right\rangle\left\langle e_{s^{\prime}}^{n}\right|$ connects two atomic states and the parameter $\delta=|\Omega|^{2} / 2 \Delta$ represents a light shift. The local polarization of the field is imprinted on each atom through the mixing angle

$$
\theta=2 \arctan \left(E_{+} / E_{-}\right),
$$

and the phases $\pm 2 k_{c} z_{n}$, which carry all the information regarding the atomic position. For atomic ensembles confined to regions much smaller than their transition wavelength this phase is irrelevant and can be absorbed into a global coupling parameter. It, however, plays a central role on extended systems [21], as we now discuss for an atomic chain.

Atoms in an ordered chain interact with one another via modes of the surrounding electromagnetic environment. Tracing out these modes under the Born and Markov approximations gives rise to a master equation for a density matrix of atomic states only [22,23]. Being interested in single-excitation dynamics, we consider a quantum trajectory evolution equivalent to the resulting master equation [24]. The state is then described by an ensemble of stochastic wave functions evolving under a Schrödinger equation with nonHermitian Hamiltonian

$$
\tilde{\mathcal{H}}=\sum_{n} \hat{\mathcal{H}}_{\mathrm{eff}}^{(n)}-\hbar \sum_{n, m=1}^{N} \sum_{s= \pm} \mathcal{K}_{s, s}^{n, m} \hat{\sigma}_{s g}^{(n)} \hat{\sigma}_{g s}^{(m)},
$$

interrupted by jump operators that drive the system into the absolute ground state when an excitation leaving the array is recorded. The interatomic coupling reads [22,23]

$$
\mathcal{K}_{s, s}^{n, m}=\frac{3 \pi \Gamma_{0}}{k_{0}} \mathbf{e}_{s}^{*} \cdot \mathbf{G}\left(z_{n}-z_{m}, \omega_{0}\right) \cdot \mathbf{e}_{s},
$$

where $\mathbf{G}\left(z_{n}-z_{m}, \omega_{0}\right)$ propagates the electric field scattered from site $n$ to site $m$, and $\mathbf{e}_{s}$ is the atomic transition polarization connecting the involved states [25]. $\Gamma_{0}$ refers to the single-atom spontaneous emission rate, and $k_{0}=\omega_{0} / c$ is the wave-vector associated with the atomic transition frequency. In the absence of the control field the Hamiltonian is diagonal in polarization indices since the scattered field does not mix different polarization components along the chain axis [26].

\section{A. Collective modes as spin waves}

The control field introduces a local rotation of the polarization that mixes different excited states along the chain. For infinite atomic chains, the system is invariant under a displacement of a lattice constant $a$ together with a rotation of an angle $k_{c} a$. This helical symmetry is represented by the operator

$$
\hat{I}\left(a, k_{c}\right)=\exp \left[\frac{i \hat{P}_{z} a+i \hat{J}_{z} k_{c} a}{\hbar}\right],
$$

which tracks the local polarization of the control field along the array, as the linear-momentum operator $\hat{P}_{z}=-i \hbar \partial_{z}$ generates displacements along the chain and the angularmomentum operator $\hat{J}_{z}=\hbar\left[\sum_{n} \hat{\sigma}_{++}^{(n)}-\hat{\sigma}_{--}^{(n)}\right]$ rotations around its axis. The helical operator commutes with the Hamiltonian and provides a basis to diagonalize it. Using this basis the eigenstates of the Hamiltonian are found to be Bloch waves of quasimomentum $k \in[-\pi / a, \pi / a)$ [20]:

$$
\begin{aligned}
|\mathrm{U}, k\rangle & =\sum_{n} e^{i k z_{n}}\left[e^{i k_{c} z_{n}} \sin \left(\alpha_{k} / 2\right) \hat{\sigma}_{-g}^{(n)}+e^{-i k_{c} z_{n}} \cos \left(\alpha_{k} / 2\right) \hat{\sigma}_{+g}^{(n)}\right]|g\rangle^{\otimes N} \\
|\mathrm{~L}, k\rangle & =\sum_{n} e^{i k z_{n}}\left[e^{i k_{c} z_{n}} \cos \left(\alpha_{k} / 2\right) \hat{\sigma}_{-g}^{(n)}-e^{-i k_{c} z_{n}} \sin \left(\alpha_{k} / 2\right) \hat{\sigma}_{+g}^{(n)}\right]|g\rangle^{\otimes N}
\end{aligned}
$$


(a) Undriven chain

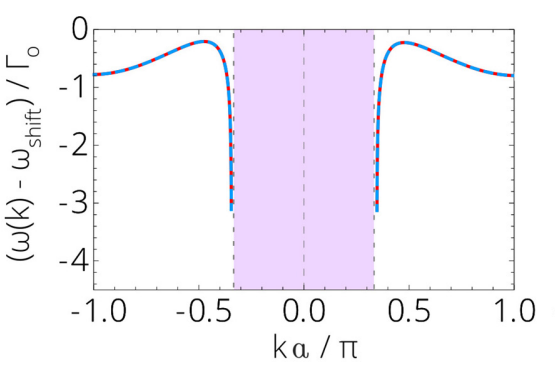

(b) Circularly polarized drive $(\theta=0)$ $-k_{c} \mathrm{a} / \pi \longleftrightarrow \mathrm{k}_{\mathrm{c}} \mathrm{a} / \pi$

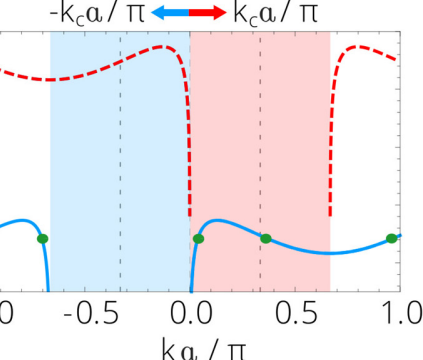

(c) Elliptically polarized drive $(\theta=\pi / 4)$

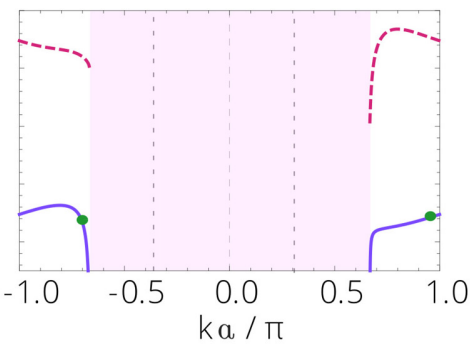

FIG. 2. Dispersion engineering by an external control field. (a) Dispersion relation of an undriven chain showing two degenerate bands that correspond to two internal degrees of freedom ( $\sigma_{ \pm}$transitions). Dispersion relation of upper (dashed line) and lower (solid) states of a chain driven by (b) circularly polarized or (c) elliptically polarized control fields. Red and blue colors represent the relative weight of the $\left|e_{+}\right\rangle$and $\left|e_{-}\right\rangle$transitions in the eigenstates, respectively. Notice that both states mix for an elliptically polarized drive. For all plots, the lattice constant is $a=\lambda_{0} / 6$ and the radiative region is shaded. In panel (a) the frequency shift is $\omega_{\text {shift }}=\omega_{0}$. In panels (b) and (c), $\omega_{\text {shift }}=\omega_{0}+(2 \Delta+\delta) / 4$ and the control field has wavelength $\lambda_{c}=\lambda_{0}$ and generates a light shift $\delta=6 \Gamma_{0}$. Green dots indicate the central frequency of the excitation plotted in Figs. 3 and 4.

where indices $\{U, L\}$ account for the internal degrees of freedom [27-29] and $\alpha_{k}$ represents the relative populations of excited states [30]. The dispersion relation $\omega_{\mathrm{U}(\mathrm{L})}$ and collective decay $\Gamma_{\mathrm{U}(\mathrm{L})}$ of upper (lower) states satisfies

$$
\begin{aligned}
\omega_{\mathrm{U}(\mathrm{L})}(k)-i \frac{1}{2} \Gamma_{\mathrm{U}(\mathrm{L})}(k)= & \frac{1}{2}\left[\Delta+\frac{1}{2} \delta-i \Gamma_{0}-\tilde{\mathcal{K}}\left(k+k_{c}\right)\right. \\
& \left.-\tilde{\mathcal{K}}\left(k-k_{c}\right)_{(-)}^{+} \frac{1}{2} \Omega_{k}\right] .
\end{aligned}
$$

In the above equation, $\tilde{\mathcal{K}}$ is the Fourier transform of Eq. (4) for atomic polarizations perpendicular to the chain axis and takes the form [4]

$$
\tilde{\mathcal{K}}(k)=\frac{3 \Gamma_{0}}{4 i} \sum_{m=1}^{3}\left(\frac{i}{a k_{0}}\right)^{m}\left[\operatorname{Li}_{m}\left(e^{i\left(k_{0}+k\right) a}\right)+\operatorname{Li}_{m}\left(e^{i\left(k_{0}-k\right) a}\right)\right],
$$

with $\mathrm{Li}_{m}$ being the polylogarithm of order $m$ [31], while $\Omega_{k}$ is the band splitting [20]

$$
\Omega_{k}=\sqrt{(\delta \sin \theta)^{2}+\left\{\delta \cos \theta+2\left[\tilde{\mathcal{K}}\left(k+k_{c}\right)-\tilde{\mathcal{K}}\left(k-k_{c}\right)\right]\right\}^{2}} .
$$

\section{B. Guided modes and dispersion engineering}

For $a<\lambda_{0} / 2$, subradiant states with $\Gamma_{\mathrm{U}(\mathrm{L})}(k)=0$ emerge. These states are identified as guided modes of the atomic waveguide and their dispersion relation is plotted in Fig. 2 for different control field values. Subradiant states lie beyond the light line (their wave vectors satisfy $|k|>k_{0}$ ) and cannot decay radiatively due to energy-momentum mismatch. This is readily seen in Fig. 2(a), where, in the absence of a control field, the guided modes associated with each state $\left|e_{ \pm}\right\rangle$are degenerate. One can understand guided modes as providing a transparency window for photons to travel along the array until scattered out at the edges. Due to the near-field dipole-dipole interaction, the transparency bandwidth scales as $\sim \Gamma_{0} /\left(k_{0} a\right)^{3}$.

The effect of the control field is exemplified in Figs. 2(b) and 2(c). The field breaks the degeneracy between excited states and introduces a relative phase between $|U, k\rangle$ and $|L, k\rangle$, which displaces the center of the light line for each band by $\pm k_{c}$. For circular polarization $[\theta=0$, Fig. 2(b)] there is no mixing between $\left|e_{ \pm}\right\rangle$states. The dispersion relation is identical to that of an undriven chain, except for a frequency shift and a displacement in quasimomentum (a transitiondependent shift from the origin). For elliptical polarizations $[\theta \neq 0$, Fig. 2(c)], internal and external degrees of freedom couple, thus mixing $\left|e_{+}^{n}\right\rangle$ and $\left|e_{-}^{m}\right\rangle$ states [see Eqs. (6)].

The angle $\theta$ is controlled by the ratio between $E_{ \pm}$amplitudes and determines the state mixing. The control field then governs the dispersion relation, allowing us to modify the bandwidth (by approaching $\theta=\pi / 2$ and reducing the coupling between neighboring sites thus causing the bands to flatten) or to make the system directional [32], for example. It also determines, indirectly, the group velocity of an excitation that propagates along the chain as a spin wave.

\section{TO TRAP AND RELEASE A TRAVELING EXCITATION}

The group velocity can then be changed mid-flight using a time-varying control field. Consider an excitation with central frequency inside the lower band $\omega_{\mathrm{L}}$ and initial group velocity $v_{\mathrm{L}}=\partial \omega_{\mathrm{L}} / \partial k$. Adiabatic changes [33-35] of the mixing angle induce a coherent, reversible transfer of this excitation from states $\left|e_{+}\right\rangle$to $\left|e_{-}\right\rangle$, while keeping its central quasimomentum unperturbed. The transfer changes the group velocity through

$$
\dot{v}_{\mathrm{L}} \propto \dot{\theta} \sin \theta \partial_{k}\left[\tilde{\mathcal{K}}\left(k+k_{c}\right)-\tilde{\mathcal{K}}\left(k-k_{c}\right)\right] .
$$

This dynamical control can be used to trap and release a pulse as it propagates along the chain. As an example, we simulate numerically an excitation propagating along a chain of $N=200$ sites. The excitation is created by an external pulse described by an additional term $\mathcal{H}_{\text {drive }}=\hbar \mathcal{E}(t)\left(\hat{\sigma}_{-g}^{(1)}+\right.$ $\left.\hat{\sigma}_{+g}^{(1)}\right)+$ H.c. in Eq. (3). The pulse has a Gaussian temporal profile

$$
\mathcal{E}(t)=\frac{\sqrt{\pi}}{\tau} e^{-i \Delta_{p} t} \exp \left[-\left(\frac{t-t_{o}}{\tau}\right)^{2}\right] \mathcal{E}_{o}
$$

centered at $t_{o}=50 \Gamma_{0}^{-1}$ with a low intensity to constrain the dynamics to the single-excitation manifold $\left(\tau=12 \Gamma_{0}^{-1}\right.$ and $\mathcal{E}_{o}=0.35$ ). It is detuned from the atomic transition by $\Delta_{p}=-3.5 \Gamma_{0}$ to populate the lower band [see green dots in Fig. 2(b)] and is considered to enter through the side of the chain where the spatial overlap to subradiant modes is 

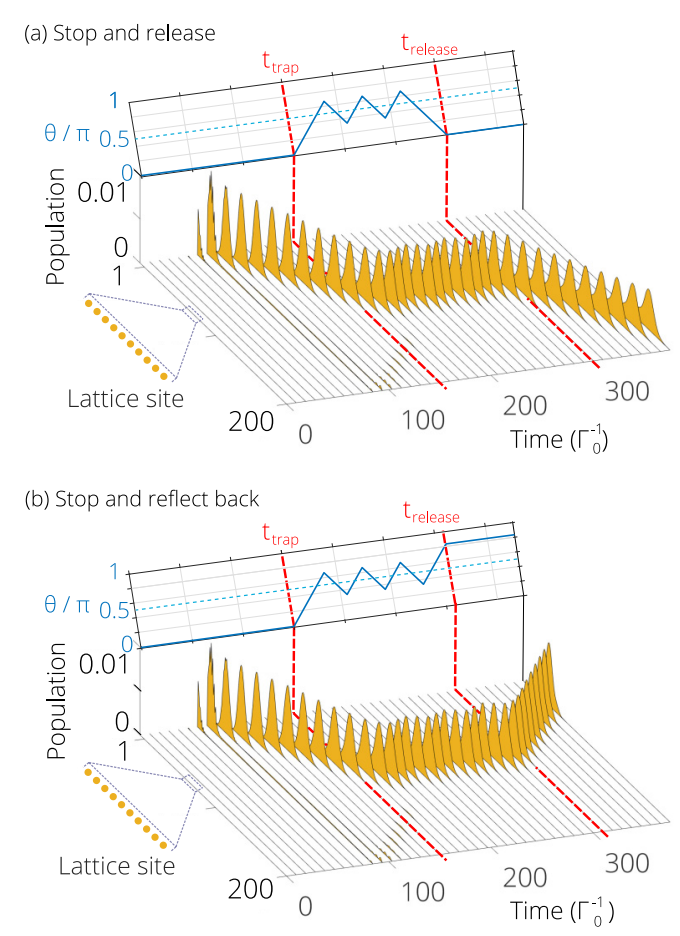

FIG. 3. Dynamical trapping of an excitation inside a chain of $N=200$ atoms before being (a) released or (b) reflected. The excited-state population of each site (yellow distribution) and mixing angle (blue line) are shown as a function of time for a chain whose parameters follow from Fig. 2(b).

maximized. Figure 3 shows the resulting spin wave as a function of time and lattice site. The evolution is conditioned to a measurement record that marks no excitations leaving the array. The chain is initially prepared in its ground state and is driven by a circularly polarized control field $(\theta=0)$ to guarantee a large bandwidth and low dispersion. The spinwave propagates freely along this chain before the control field is adiabatically changed until it becomes linearly polarized $(\theta=\pi / 2)$, bringing the wave near to a standstill. The mixing angle is then varied to oscillate around $\theta=\pi / 2$ for three cycles before it is released $[\theta=0$, Fig. 3(a)] or reflected back $[\theta=0.8 \pi$, Fig. 3(b) $]$.

The trapping and retrieval of an excitation is limited by the decay rate and dispersion of the chain. For long but finite chains, subradiant modes couple to the electromagnetic environment at both ends of the chain-where radiative paths cannot cancel through destructive interference-and acquire a small decay rate that decreases with the site number as $N^{-3}$ [4]. This spatially localized coupling allows for both a decay-free propagation of a spin-wave inside the chain and a nonzero decay at the ends where it scatters out. We plot the population of the chain in Fig. 4(b) to exemplify this behavior. Notice the small dip at time $t \simeq 75 \Gamma_{0}^{-1}$. It occurs because the driving field also couples to fast spin waves that reach the end of the chain shortly after being excited and are scattered into free space or reflected back. Slow spin waves, by contrast, remain far from the edges at this time and are trapped without losses. Once released, the slow spin wave will eventually reach the end of the chain and scatter into a traveling light pulse [20].
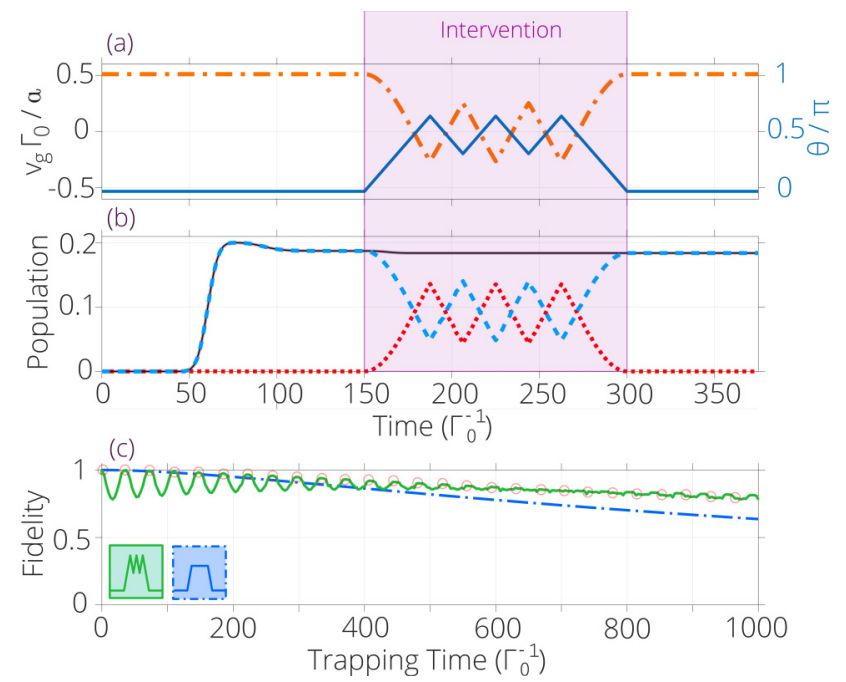

FIG. 4. Internal dynamics and dispersion control of the excitation trapped in Fig. 3(a). (a) Group velocity (orange dashed) and mixing angle (blue solid). (b) Total population of the excited state (black solid) with $\left|e_{-}\right\rangle$(blue dashed) and $\left|e_{+}\right\rangle$(red dotted) contributions. (c) Fidelity of the protocol for cyclic (solid green) and constant (dashed blue) interventions. Red circles indicate the end of a cycle.

\section{A. Range of validity of the trapping protocol}

Dispersion of the spin wave limits its trapping time, but this effect can be corrected by the control field. The dispersion relations $\omega_{\mathrm{L}}$ and $\omega_{\mathrm{U}}$ display opposite curvatures in most of the subradiant region [see solid and dashed lines in Figs. 2(b) and 2(c)]. Therefore, the phase acquired by most quasimomentum components changes from positive to negative as the ellipticity of the control field is varied. While this behavior can be exploited to trap an excitation, it can also be used to compensate for its natural dispersion. Figure 4(c) shows the fidelity of the protocol as a function of trapping time and compares the case of a cycling ellipticity to that of a constant value $(\theta=\pi / 2)$. The fidelity $\mathcal{F}(t)=\left|\left\langle\psi\left(t_{\pi / 2}\right) \mid \psi(t)\right\rangle\right|^{2}$ quantifies changes of the spin wave after it is stopped at time $t_{\pi / 2}$ and released at a time $t$ through their normalized wave functions. As the ellipticity of the control field changes, there is a partial rephasing of different quasimomentum components that reduces the dispersion.

The experimental realization of these ideas requires stringent (but achievable) conditions on the hyperfine structure of the atomic species and underlying lattice constant. It requires a $F_{g}=0$ to $F_{e}=1$ transition (found in bosonic strontium and ytterbium) and deeply subwavelength interatomic distances (recently achieved in optical lattice setups [36-38]). It also requires for similar control field and atomic resonance frequencies, imposing that $k_{c} \simeq k_{0}$. As small lattice constants give rise to increased Brillouin zones and larger bandwidths, the relative shift in quasimomentum of upper and lower bands decreases. This makes the two bands similar and makes it difficult to find regions where they display the opposite curvatures required to trap an excitation. To move past this restriction one can choose a control field that couples the excited states to a third, highly excited, state. The third state acts as a bridge between $\left|e_{ \pm}\right\rangle$while allowing for $k_{c}$ and $k_{0}$ to be independent from each other. 


\section{CONCLUSION}

Dynamical control and dispersion engineering make atomic arrays into versatile light-matter interfaces. We have shown how to manipulate the optical properties of ordered atomic media via external control fields, a task hard to achieve in conventional dielectric structures. These ideas were exploited to trap and release an excitation, opening the door to novel protocols for quantum information processing. Long trapping times may enable the realization of two-photon gates without the need for Rydberg interactions. Our proposal also allows us to engineer exotic properties, such as directional transport [39].

\section{ACKNOWLEDGMENTS}

We thank D. E. Chang, J. A. Muniz, H. Ochoa, A. Gonzalez-Tudela, R. Jáuregui, and L. A. Orozco for insightful comments and discussions. R.G.-J. and A.A.-G. acknowledge financial support by the National Science Foundation QIITAQS (Award No. 1936359).
[1] D. Barredo, V. Lienhard, S. de Léséleuc, T. Lahaye, and A. Browaeys, Synthetic three-dimensional atomic structures assembled atom by atom, Nature (London) 561, 79 (2018).

[2] R. J. Bettles, S. A. Gardiner, and C. S. Adams, Enhanced Optical Cross Section via Collective Coupling of Atomic Dipoles in a 2D Array, Phys. Rev. Lett. 116, 103602 (2016).

[3] E. Shahmoon, D. S. Wild, M. D. Lukin, and S. F. Yelin, Cooperative Resonances in Light Scattering from Two-Dimensional Atomic Arrays, Phys. Rev. Lett. 118, 113601 (2017).

[4] A. Asenjo-Garcia, M. Moreno-Cardoner, A. Albrecht, H. J. Kimble, D. E. Chang, Exponential Improvement in Photon Storage Fidelities Using Subradiance and "Selective Radiance" in Atomic Arrays, Phys. Rev. X 7, 031024 (2017).

[5] S-M. Yoo and J. Javanainen, Light reflection and transmission in planar lattices of cold atoms, Opt. Express 28, 9764 (2020).

[6] J. Rui, D. Wei, A. Rubio-Abadal, S. Hollerith, J. Zeiher, D. M. Stamper-Kurn, C. Gross, and I. Bloch, A subradiant optical mirror formed by a single structured atomic layer, Nature (London) $\mathbf{5 8 3}, 369$ (2020).

[7] J. A. Needham, I. Lesanovsky, and B. Olmos, Subradianceprotected excitation transport, New J. Phys. 21, 073061 (2019).

[8] S. J. Masson and A. Asenjo-Garcia, Atomic-waveguide quantum electrodynamics, Phys. Rev. Research 2, 043213 (2020).

[9] R. Holzinger, D. Plankensteiner, L. Ostermann, and H. Ritsch, Nanoscale Coherent Light Source, Phys. Rev. Lett. 124, 253603 (2020).

[10] K.-J. Boller, A. Imamoglu, and S. E. Harris, Observation of Electromagnetically Induced Transparency, Phys. Rev. Lett. 66, 2593 (1991).

[11] J. E. Field, K. H. Hahn, and S. E. Harris, Observation of Electromagnetically Induced Transparency in Collisionally Broadened Lead Vapor, Phys. Rev. Lett. 67, 3062 (1991).

[12] S. E. Harris, J. E. Field, and A. Kasapi, Dispersive properties of electromagnetically induced transparency, Phys. Rev. A 46, R29 (1992).

[13] L. V. Hau, S. E. Harris, Z. Dutton, and C. H. Behroozi, Light speed reduction to 17 metres per second in an ultracold atomic gas, Nature (London) 397, 594 (1999).

[14] G. Juzeliunas, Microscopic theory of quantization of radiation in molecular dielectrics: Normal-mode representation of operators for local and averaged (macroscopic) fields, Phys. Rev. A 53, 3543 (1996).

[15] G. Juzeliunas and H. J. Carmichael, Systematic formulation of slow polaritons in atomic gases, Phys. Rev. A 65, 021601(R) (2002).
[16] M. Fleischhauer and M. D. Lukin, Quantum memory for photons: Dark-state polaritons, Phys. Rev. A 65, 022314 (2002).

[17] J. Dalibard and C. Cohen-Tannoudji, Laser cooling below the Doppler limit by polarization gradients: Simple theoretical models, J. Opt. Soc. Am. B 6, 2023 (1989).

[18] A. Messiah, Quantum Mechanics (North-Holland, Amsterdam, 1961), Vol. II, Chaps. XVI and XVII.

[19] J. Mitroy, M. S. Safronova, and C. W. Clark, Theory and applications of atomic and ionic polarizabilities, J. Phys. B: At., Mol. Opt. Phys. 43, 202001 (2010).

[20] See Supplemental Material at http://link.aps.org/supplemental/ 10.1103/PhysRevResearch.4.013080 for a detailed derivation of the master equation (3), its solutions in the infinite case limit and departure of the single-photon scenario. A description of the spatial and temporal profiles of the electromagnetic field is also given

[21] R. H. Lehmberg, Radiation from an $N$-atom system. I. General formalism, Phys. Rev. A 2, 883 (1970).

[22] M. Gross and S. Haroche, Superradiance: An essay on the theory of collective spontaneous emission, Phys. Rep. 93, 301 (1982).

[23] H. J. Carmichael and K. Kim, A quantum trajectory unraveling of the superradiance master equation, Opt. Commun. 179, 417 (1999).

[24] H. J. Carmichael, Statistical Methods in Quantum Optics 2 (Springer-Verlag Berlin, Heidelberg, 2008), Chap. 17.

[25] The propagator takes the explicit form

$$
\mathbf{G}\left(\mathbf{r}, \omega_{0}\right)=\frac{k_{0}}{4 \pi} \frac{e^{i \xi}}{\xi^{3}}\left[\left(\xi^{2}+i \xi-1\right) \mathbb{1}-\left(\xi^{2}+3 i \xi-3\right) \frac{\mathbf{r} \otimes \mathbf{r}}{r^{2}}\right],
$$

with $\xi=k_{0} r$ as found in Refs. [4,22,23].

[26] A. Asenjo-Garcia, H. J. Kimble, and D. E. Chang, Optical waveguiding by atomic entanglement in multilevel atom arrays, Proc. Natl. Acad. Sci. USA 116, 25503 (2019).

[27] Y. Castin and J. Dalibard, Quantization of atomic motion in optical molasses, Europhys. Lett. 14, 761 (1991).

[28] P. Marte, R. Dum, R. Taïeb, P. D. Lett, and P. Zoller, Quantum Wave Function Simulation of the Resonance Fluorescence Spectrum From One-Dimensional Optical Molasses, Phys. Rev. Lett. 71, 1335 (1993).

[29] W. Ren and H. J. Carmichael, Spontaneous emission in a standing-wave cavity: Quantum-mechanical center-of-mass motion, Phys. Rev. A 51, 752 (1995).

[30] The angle $\alpha_{k}$ is defined by

$$
\tan \alpha_{k}=\frac{\delta \sin \theta}{\delta \cos \theta+2\left[\tilde{\mathcal{K}}\left(k+k_{c}\right)-\tilde{\mathcal{K}}\left(k-k_{c}\right)\right]} .
$$


[31] M. Abramowitz and I. A. Stegun, Handbook of Mathematical Functions with Formulas, Graphs, and Mathematical Tables (Dover, New York, 1972).

[32] Y. Hadad and B. Z. Steinberg, Magnetized Spiral Chains of Plasmonic Ellipsoids for One-Way Optical Waveguides, Phys. Rev. Lett. 105, 233904 (2010).

[33] M. Ben Dahan, E. Peik, J. Reichel, Y. Castin, and C. Salomon, Bloch Oscillations of Atoms in an Optical Potential, Phys. Rev. Lett. 76, 4508 (1996).

[34] E. Peik, M. Ben Dahan, I. Bouchoule, Y. Castin, and C. Salomon, Bloch oscillations of atoms, adiabatic rapid passage, and monokinetic atomic beams, Phys. Rev. A 55, 2989 (1997).

[35] J. Dalibard, F. Gerbier, G. Juzeliunas, and P. Ohberg, Colloquium: Artificial gauge potentials for neutral atoms, Rev. Mod. Phys. 83, 1523 (2011).
[36] Y. Wang, S. Subhankar, P. Bienias, M. Lacki, T-C. Tsui, M. A. Baranov, A. V. Gorshkov, P. Zoller, J. V. Porto, and S. L. Rolston, Dark State Optical Lattice with a Subwavelength Spatial Structure, Phys. Rev. Lett. 120, 083601 (2018).

[37] R. P. Anderson, D. Trypogeorgos, A. Valdés-Curiel, Q.-Y. Liang, J. Tao, M. Zhao, T. Andrijauskas, G. Juzeliunas, and I. B. Spielman, Realization of a deeply subwavelength adiabatic optical lattice, Phys. Rev. Research 2, 013149 (2020).

[38] T-C. Tsui, Y. Wang, S. Subhankar, J. V. Porto, and S. L. Rolston, Realization of a stroboscopic optical lattice for cold atoms with subwavelength spacing, Phys. Rev. A 101, 041603(R) (2020).

[39] R. Gutiérrez-Jáuregui and A. Asenjo-Garcia, Directional Transport Along an Atomic Chain (submitted for publication), arXiv:2111.06734. 